\title{
Incidence of Acute Undifferentiated Fevers - Kadapa District- Analytical Study
}

\author{
Authors \\ M. Satyanarayana Raju ${ }^{1}$, M.S. Khaja Mohiddin ${ }^{2}$ \\ ${ }^{1}$ Assistant Professor, Dept. of Community Medicine, GVP IHC \& MT, Visakhapatnam, AP \\ ${ }^{2}$ District Epidemiologist, IDSP O/o: DM \& HO, Kadapa \\ Email:msraju49@gmail.com
}

\begin{abstract}
Acute fevers mentioned in the core conditions of the Integrated Diseases Surveillance Project (IDSP) i.e. Malaria, Dengue, ARI and typhoid are under regular surveillance in Kadapa district. Generally in any given year ten to eleven percent of the total out patients' attendance consists of fevers. The medical officer is expected to examine the patients and make presumptive diagnosis basing on the clinical findings and advise the clinical laboratory tests for confirmation of diagnosis. The laboratories tests are basic in nature, conducted at the PHC level i.e. blood smear examination for malaria parasite, serological tests for rapid diagnosis of Dengue, Typhoid etc. Fevers were analyzed month wise, year wise and found that 18 to 20 percent of the fevers did not show positive for basic tests conducted at PHC like blood smear examination for Malaria, Typhoid dot for enteric fevers, Rapid diagnostic tests for dengue etc and remain as (2) undifferentiated fevers. When they were subjected to statistical analysis they start rising from the month of June just as seasonal fevers i.e. the other important observation was incidence of malaria and dengue were found to be low in kadapa district ${ }^{(1)}$ Though Andhra Pradesh falls under category 2 of the pre elimination phase, identification malaria cases is low in kadapa district (endemic for malaria) with many false negatives. Supporting the above observation incidence of malaria and ${ }^{(3)}$ dengue are low in kadapa district though it is endemic for malaria. A thorough analysis is done to identify the causes of false negatives and to take rectification measures.

Keywords: Undifferentiated fevers, Dengue, Malaria, Kadapa.
\end{abstract}

\section{Introduction}

YSR District is centrally located and well connected with four districts of Rayalaseema. It is also the land locked district within the State and lies approximately between $13043^{\prime}$ to 150 14' North latitudes and $77055^{\prime}$ to 790 29' of East longitudes. The total area of the district is 15,359 square kilometers and forest area is 5,017.33 Sq. $\mathrm{Km}$. While conducting health profile study of kadapa district in 2017 and reviewing the core conditions in ${ }^{(4)}$ Integrated Disease Surveillance Program (IDSP) including acute fevers, Malaria and Dengue. It was found that large proportion of fever cases were undiagnosed and mentioned as undifferentiated fevers.

Disease surveillance was a concept already in existence and defined as the continuous scrutiny of all aspects of occurrence and spread of diseases that are pertinent to effective control. Surveillance goes beyond the passive reporting of cases. It 
includes laboratory conformation of presumptive diagnosis, finding out source of infection, routes of transmission, identification of all cases and susceptible contacts and still others who are at risk in order to finally prevent further spread of disease. During the present study analysis among fevers, significant number is undiagnosed and mentioned as undifferentiated fevers.

Kadapa has 3 revenue divisions and 51 mandals with a population of 2882469 . Among population, $82 \%$ are below poverty line. There are 72 PHCs and 448 SUB centers. Basic clinical lab tests for diagnosis of common ailments particularly for confirmation of Malaria, Typhoid and ${ }^{(5)}$ dengue are available at PHC. There is one municipal corporation and 8 municipalities fall under urban and peri-urban areas of kadapa district, contributing large no of fever cases due to deficient water management including improper water storage practices favorable for mosquito breeding resulting in high transmission.

\section{Material and Methods}

Kadapa District is very hot place and falls in to scarce rain fall area. There is one municipal corporation and 8 municipalities. The density of population in the district is 169 per sq/ $/ \mathrm{km}$. The district has a tropical wet and dry climate characterized by year round high temperatures with humidity. During this time temperatures range from a minimum of $34{ }^{\circ} \mathrm{C}$ and humidity is around $75 \%$. Monsoon season bring substantial rain to the area.

Secondary data was obtained from DM\&HO of Kadapa district for the last six years (2012-2017). The author of the present article himself worked as DM\&HO kadapa for entire year 2015. A Retrospective study was conducted to know about the un-diagnosed fevers mentioned as undifferentiated fevers in each year. Incidence of malaria and dengue were obtained for the last 6years (2012 -2017). Month wise distribution of undifferentiated fevers was studied. The incidence of seasonal diseases i.e. malaria, dengue were also studied month wise along with undifferentiated fevers. Distribution of Malaria, dengue and undifferentiated fevers are similar i.e. rise in incidence during man soon period i.e. from June to November and hence it is inferred that, significant cases of malaria and dengue could not be diagnosed resulted in false negatives.

Diagnostic tools: All primary health centers were provided with microscopy and necessary staining material. Qualified technicians are posted. ASHA and other volunteer workers are provided with RDTs. For diagnosis of Dengue in addition to hematological parameters (platelet count and hematocrit) at PHC level commercial rapid format serological test kits(RDT) ${ }^{(6)}$ for anti-dengue $\operatorname{IgM}$ and igG anti bodies have become available from the past few years.

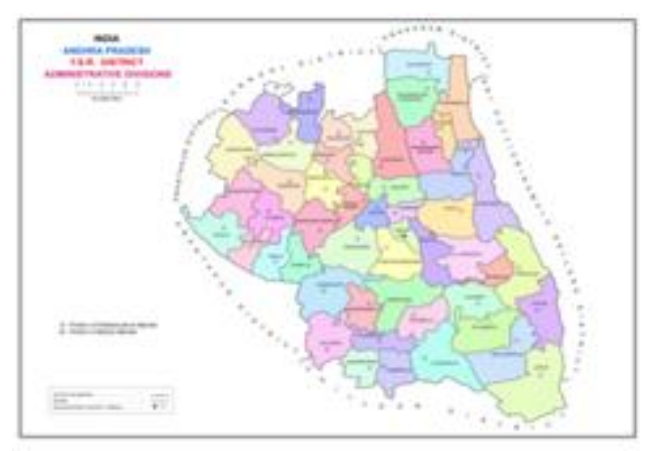

\section{Results}

Table-1: Country wide malaria surveillance data 2000-2015

\begin{tabular}{|c|c|c|c|c|c|c|}
\hline Year & $\begin{array}{c}\text { Blood } \\
\text { exam in } \\
\text { million }\end{array}$ & $\begin{array}{c}\text { Positive } \\
\text { cases in } \\
\text { millions }\end{array}$ & PF\% & ABER & API & SPR \\
\hline 2000 & 86.79 & 2.03 & 51.5 & 8.9 & 2.1 & 2.3 \\
\hline 2010 & 108.68 & 1.6 & 52.2 & 9.3 & 1.4 & 1.5 \\
\hline 2011 & 10897 & 1.31 & 50.3 & 8.9 & 1.1 & 1.2 \\
\hline 2012 & 109,03 & 1.07 & 50 & 9 & 0.9 & 1 \\
\hline 2013 & 113.11 & 0.88 & 52.6 & 9.3 & 0.7 & 0.8 \\
\hline 2014 & 124.07 & 1.1 & 65.6 & 10.1 & 0.9 & 0.9 \\
\hline 2015 & 118.47 & 1.13 & 67.1 & 9.6 & 0.9 & 0.7 \\
\hline
\end{tabular}

Table-2: Kadapa district wise malaria surveillance data (2012-2017)

\begin{tabular}{|c|c|c|c|c|c|c|}
\hline Year & $\begin{array}{c}\text { Blood } \\
\text { examination in } \\
\text { thousands }\end{array}$ & $\begin{array}{c}\text { Positive } \\
\text { cases in } \\
\text { hundreds }\end{array}$ & PF \% & ABER & API & SPR \\
\hline 2012 & 247733 & 623 & 93 & 8.5 & 0.21 & 0.25 \\
\hline 2013 & 225160 & 205 & 34 & 7.8 & 0.07 & 0.09 \\
\hline 2014 & 285189 & 316 & 54 & 9.8 & 0.1 & 0.1 \\
\hline 2015 & 334061 & 292 & 49 & 11.5 & 0.1 & 0.08 \\
\hline 2016 & 349611 & 661 & 90 & 12.1 & 0.2 & 0.19 \\
\hline 2017 & 353547 & 601 & 48 & 12.2 & 0.21 & 0.16 \\
\hline
\end{tabular}




\section{JMSCR Vol||06||Issue||03||Page 894-898||March}

Figure-2: Distribution of undifferentiated fevers month wise from 2014 to 2017

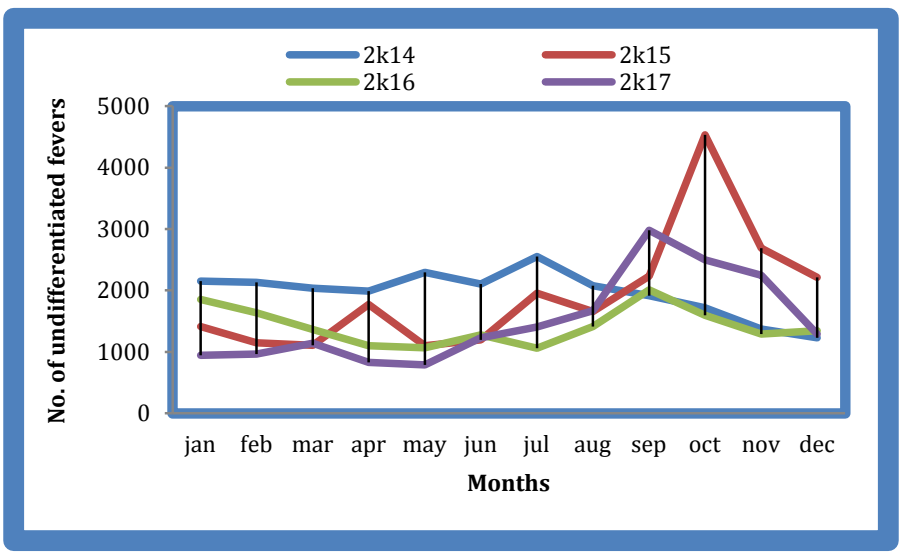

Figure-3: Distribution of malaria cases month wise from 2014 to 2017

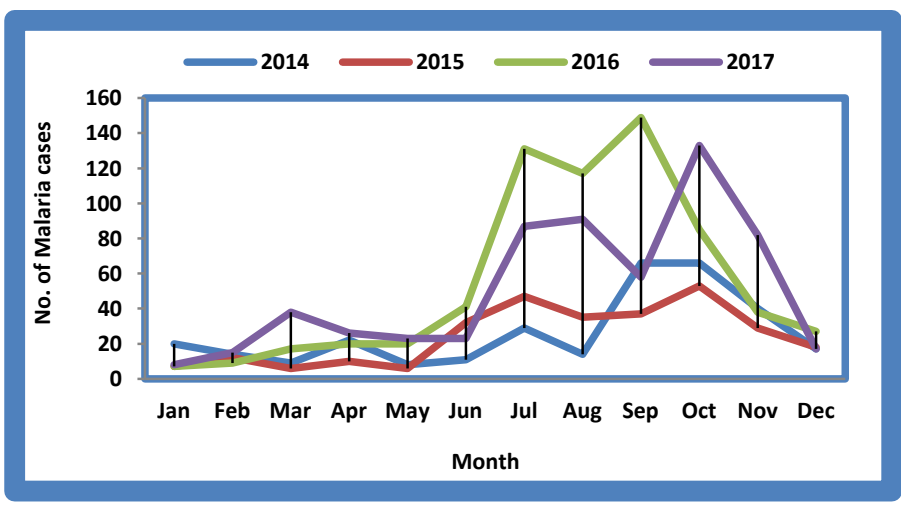

Figure-4: Distribution of dengue fevers month wise from 2014 to 2017

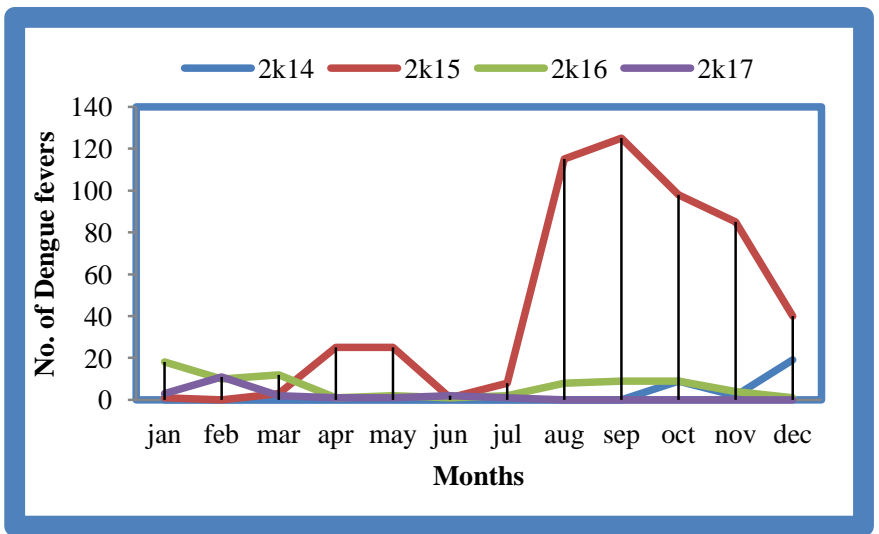

Figure-5: (1) Monthly distribution pattern of malaria, dengue ${ }^{(7)}$ and un-differentiated fevers for the years 2014 to 2017 are similar showing all the three categories are seasonal fevers associated with rainy season.

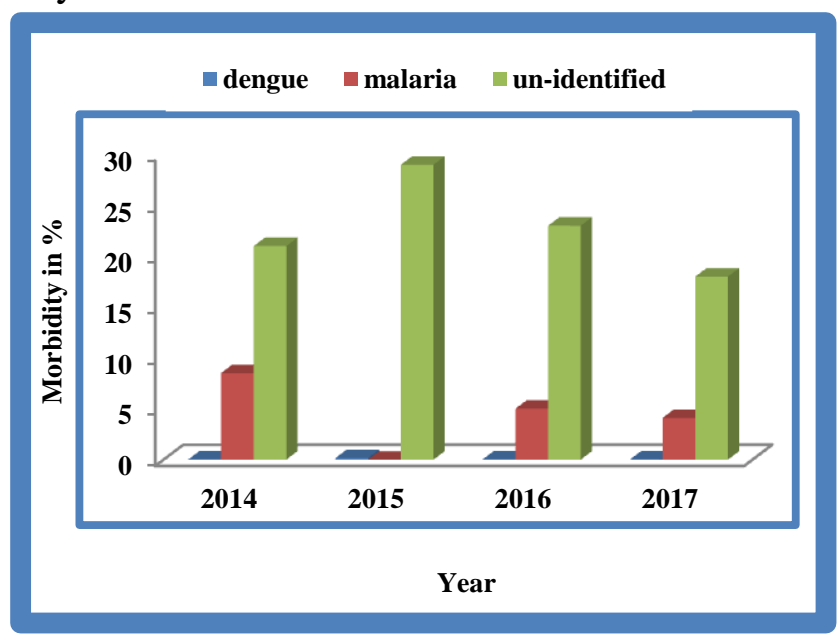

Table-3: API in Country and Kadapa District wise information from 2012 to 2017

\begin{tabular}{|l|c|c|}
\hline \multirow{2}{*}{ Year } & \multicolumn{2}{|c|}{ API } \\
\cline { 2 - 3 } & INDIA & KADAPA \\
\hline $2 \mathrm{k} 12$ & 0.9 & 0.21 \\
\hline $2 \mathrm{k} 13$ & 0.7 & 0.07 \\
\hline $2 \mathrm{k} 14$ & 0.9 & 0.1 \\
\hline $2 \mathrm{k} 15$ & 0.9 & 0.1 \\
\hline $2 \mathrm{k} 16$ & & 0.2 \\
\hline $2 \mathrm{k} 17$ & & 0.21 \\
\hline
\end{tabular}

Figure-6: API in Country and Kadapa District wise information from 2012 to 2017

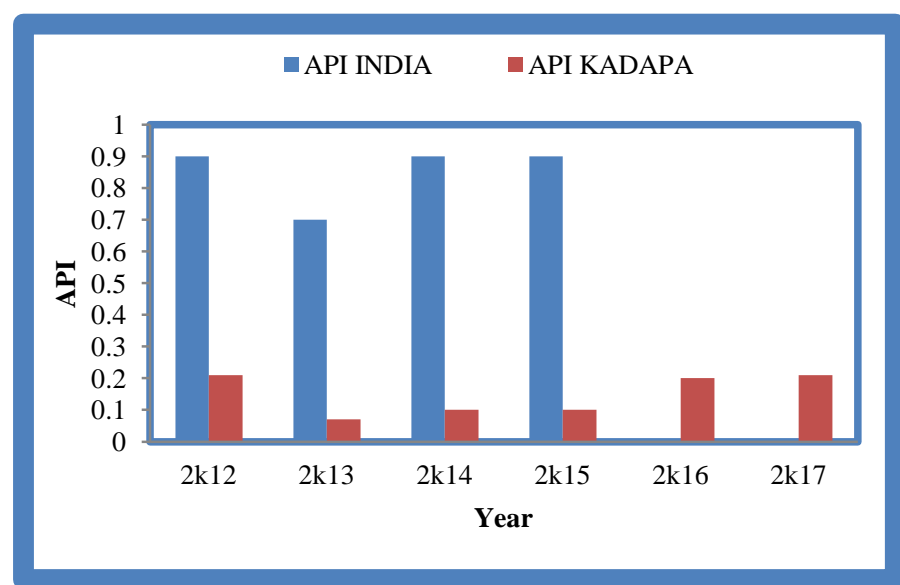


Table-4: SPR in Country and Kadapa District wise information from 2012 to 2017

\begin{tabular}{|c|c|c|}
\hline \multirow{2}{*}{ Year } & \multicolumn{2}{|c|}{ SPR } \\
\cline { 2 - 3 } & INDIA & KADAPA \\
\hline 2012 & 1 & 0.25 \\
\hline 2013 & 0.8 & 0.09 \\
\hline 2014 & 0.9 & 0.1 \\
\hline 2015 & 0.7 & 0.08 \\
\hline 2016 & & 0.19 \\
\hline 2017 & & 0.16 \\
\hline
\end{tabular}

Figure-7: SPR in Country and Kadapa District wise information from 2012 to 2017

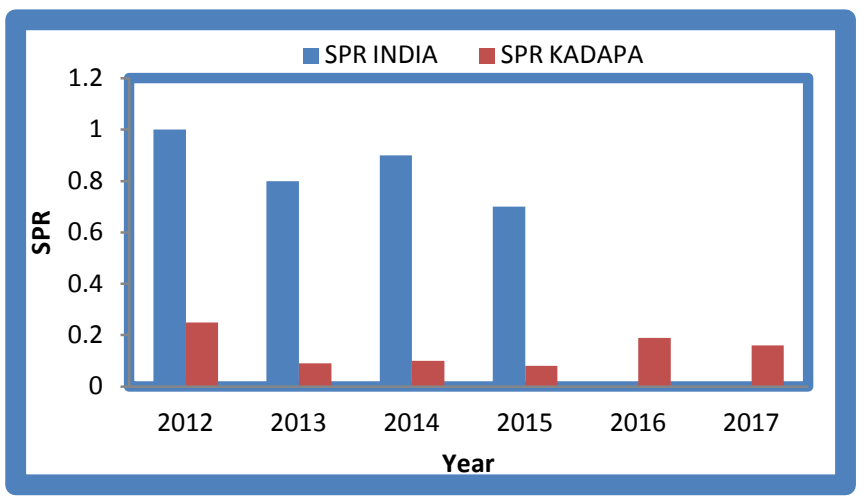

(2) Annual Parasite incidence (API) for kadapa district is low when compared with national average during the same period.

(3)Slide positivity rate is also very low. The two most important surveillance data indicate low quality of malaria work in kadapa district ${ }^{(8)}$ Malaria cases were not detected resulting in false negatives. Likewise dengue also not effectively screened and large number of cases was identified as undifferentiated fevers.

\section{Discussion}

Malaria and dengue both are vector borne diseases and closely related to rainy season. In our present study large no of fevers could not be diagnosed and identified as un-differentiated fevers. Month wise and year wise incidence of malaria, dengue and undifferentiated fevers showed similar distribution confirming significant association. Large number of un-differentiated fevers may be false negatives for both malaria and dengue. Though dengue fever is self-limiting to a large extent small percentage cases may manifest as ${ }^{(9)}$ DHF /DSS resulting in high morbidity. Untreated malaria cases ${ }^{(10)}$ may have recurrence leading to high morbidity and in both the cases there may be high transmission. If the false negative cases visit the private hospitals in Kadapa or neighboring states Chennai and Bengalure, they may have to meet out of pocket expenses and financial loss.

All the PHCs are provided with microscope, necessary staining materials and qualified technician. RDTs are now the established method of choice for malaria diagnosis

Rapid diagnostic tests are based on the detection of circulating parasite antigen with a simple dipstick format. Several types of RDTs are available. RDTs are produced by different companies, so there may be differences in the contents and in the manner in which the test is done, the user's manual should always be reed properly to avoid false negative results. A number of commercial rapid format serological test kits for anti dengue IgM and IgG antibodies have become available in the past few years. Some of these producing results within 15 minutes. Unfortunately accuracy of most of these tests is uncertain since they have not yet been properly (11) (12) validated. Hence the gold standard tests for malaria and dengue infection are peripheral blood smear examination under microscopy for malaria parasite and haematolological parameters such as platelet count and hematocrit are important part of the diagnosis for both the diseases respectively.

\section{Conclusion}

Field level health functionaries ASHA and voluntary workers need (13) capacity building trainings to use the RDT kits in interior habitations and villages for correct interpretation. All fever cases with negative results shall have close follow up till fever subsides or for further review of the case by PHC medical officer. There shall be accountability for each and every fever case during rainy season particularly, by entrusting to ANM/Health Assistant in the area of their jurisdiction for complete course of medication in case of malaria, and observe the dengue/suspected dengue case to detect the complications at the earliest to take remedial measures. 


\section{References}

1. K.Park. Preventive and Social Medicine, Twenty-Fourth Edition Ed. Jabalpur: M/S Banarasidas Bhanot; 2017.

2. Kristine Morch, Anand Manohara, Sara Crandy, Novin Chacko, Gerardo Alvarez Uria,Suvama Patil,Anil Henry etal. Acute undiferentiated fever in India: a multi centre study of aetiology and diagnostic accuracy. BMC infectious Diseases 2017; 17(PMC5628453): .

3. Kundavaram Paul Prabhaar Abhilash, Janathan Arul Jeevan, Subhankar Mitra, Nirvin Paul, Thimori Plani Murugam, Ajay Ranga Raj etal. Acute Undifferentiated febrile illness in patiets presenting to a Tertiary care hospital in South India: clinical spectrum and outcome.. Journal of Global Infectious Disease 2016; 8(4): .

4. DR Srivastav, S Venkatesh, S.Pandey, Ravi Sankar, D.S.Pillai. Completness and Timeliness of Reporting Under Itigated Disease Surveillance Project in rural Surveillance Unit of Nanital District of Uttarakhand, India.. Indian Journal of Preventive and Scial Medicine. 2009; 40(3 and 4): .

5. James A. Potts and Alan L.Rothman. clinical and Laboratory features that distinuish dengue from other febrile illnesses in endemic population. Tropical Medicine and International Journal 2008; 13(11): .

6. Mitra S , Choudhari R, Nori H, Abhilash K $\mathrm{P}$, Jayaseelan V. etal. Comprehensive evaluation of Validity and cost-benifit analysis of rapid diagnostic tests(RDT) Kits in Diagnosis of dengue infection using composit reference criteria: A crosssectional study from South India.. PubMeD-Journal of Vector Borne Diseses 2016; 53(1 30-6): .

7. S. Wongkoon, $\mathrm{M}$ jaroensutasinee and $\mathrm{k}$. Jaroensutasinee.. Distribution of, Seassonal Variation \& Dengue transmission prediction in Sisaket, Thailand.. IJMR Indian Journal of Medical Research. 2013; 138(3): .

8. Aswani Kumar,Neena Valecha,Tanu Jain and Aditya P.Dash.. Defining and Defending the Intolerable Burden of Malaria III: Progress and Prospective, American Society of Tropical Medicine and Hygiene, 2007; 77(6): .

9. Agarwal A,Chandra J,Anela .S,Patwari A.K,Dutta A.K. An Epidemic of Dengue hemorrhagic fever and Dengue shock syndrome in children in Delhi.. NCBI Pub Med 1998; 35(8): .

10. Aswani KUMAR, Laura Chery,Chinmoy Biswas,Nagesh Dubhashi, Prafulla Dutta,Virendra Kumar Dua et al. Malaria in South Asia: prevention and control. HHS Public Access 2012; 121(3): .

11. S. Vyas, B. Puwar, V. Patel, G. Bhatt, S. Kulkarni and M. Fancy.. Study on Validity of a rapid diagnostic test kits versus light microscopy for Malaria. Eastern Mediterranian Health Journal 2014; 20(4):

12. Scott R Fry,Michelle Meyer,Mathew G Sample,Cmeron p .Simmons, Shamala Devi Sekaran,Johnny X Hvang. A Diagnostic Sensitivity of Dengue Rapid Test Assay is Significantly Enhanced by Using a combined Antigen and Antibody Testing Approach. PLOS NEGLECTED TROPICAL DISEASES A peer-Reviewed Open Access Journal 2011; 5(6): .

13. John C. Reeder and Jamie A guth.. What have we learned from 40 years of supporting Research and capacity building ?. PLOS NEGLECTED TROPICAL DISEASES A peer-Reviewed Open Access Journal 2015; 9(1): . 